\title{
Sistema para Reabilitação Motora e Análise Compensatória pós Acidente Vascular Encefálico
}

\author{
Gabriel Cyrino \\ gabrielcyrino@ufu.br \\ Faculdade de Engenharia Elétrica \\ Universidade Federal de Uberlândia \\ Uberlândia, Brasil \\ Alexandre Cardoso \\ alexandre@ufu.br \\ Faculdade de Engenharia Elétrica \\ Universidade Federal de Uberlândia \\ Uberlândia, Brasil
}

\author{
Najara Zago \\ najara.zago@ufu.br \\ Faculdade de Engenharia Elétrica \\ Universidade Federal de Uberlândia \\ Uberlândia, Brasil \\ Alcimar Soares \\ alcimar@ufu.br \\ Faculdade de Engenharia Elétrica \\ Universidade Federal de Uberlândia \\ Uberlândia, Brasil
}

\author{
Roberta Aramaki \\ roberta.aramaki@ufu.br \\ Faculdade de Engenharia Biomédica \\ Universidade Federal de Uberlândia \\ Uberlândia, Brasil \\ Edgard Lamounier \\ lamounier@ufu.br \\ Faculdade de Engenharia Elétrica \\ Universidade Federal de Uberlândia \\ Uberlândia, Brasil
}

\section{RESUMO}

O Acidente Vascular Encefálico é uma das doenças mais comuns que levam ao comprometimento da destreza dos membros superiores. Novas abordagens terapêuticas, como ambientes virtuais, têm se preocupado com o aprimoramento avaliativo de protocolos individualizados, que integram a reabilitação sensorial para uma maior recuperação funcional de indivíduos. Porém, em resposta a perda funcional de um membro, é comum o aprendizado de formas compensatórias de movimento. Esta proposta aborda um sistema composto por um ambiente virtual e um dispositivo robótico, altamente personalizáveis com o objetivo de auxiliar na reabilitação motora de pacientes com hemiparesia nos membros superiores, decorrente de um Acidente Vascular Encefálico, bem como analisar sua dinâmica de movimento através de um modelo biomecânico.

\section{PALAVRAS-CHAVE}

Acidente Vascular Encefálico, Ambiente Virtual, Dispositivo Robótico, Reabilitação Neurológica, Compensação.

\section{INTRODUÇÃO}

O Acidente Vascular Encefálico (AVE) é uma síndrome caracterizada pelo início súbito de sinais clínicos de déficit neurológico ocasionada por um distúrbio da circulação sanguínea encefálica que compromete a função do encéfalo de forma focal ou global [2] Segundo dados do Ministério da Saúde, o AVE representa a segunda principal causa de mortalidade e a primeira causa de incapacidade no Brasil, com incidência de mais de 216 mil casos anuais. [1].

Dentre os sistemas acometidos pós AVE, o sistema motor tende a ser o mais comprometido, resultando em perda ou limitação da função muscular, do controle do movimento e limitação da mobilidade. Os déficits neurológicos decorrentes do AVE comprometem a funcionalidade do indivíduo, podendo de acordo com [18], manifestar-se em $80 \%$ dos casos como hemiparesia, sendo esta, a manifestação mais comum pós AVE. A hemiparesia caracteriza-se pela inabilidade funcional do membro, devido à perda parcial de força [3].

Nos primeiros momentos após o evento (fase aguda), algumas funções podem ser recuperadas à medida que, por exemplo, o edema encefálico é reduzido, e graças ao processo de reestruturação neuroplástica precoce [13]. Porém, apesar da grande quantidade de programas de reabilitação existentes, a recuperação completa da função motora, especialmente de membros superiores, através de terapias convencionais, ainda é um desafio [6].

Diante disso, novas abordagens terapêuticas cada vez mais têm se preocupado com o aprimoramento avaliativo sensoriomotor e de protocolos individualizados, que integram a reabilitação sensorial para uma maior recuperação funcional destes indivíduos [6]. Dentre tais abordagens, estão os jogos sérios e ambientes virtuais, que podem promover a neuroplasticidade ao fornecer um treinamento orientado a tarefa, com altas repetições e ajuste de parâmetros, tais como tempo da terapia, intensidade e desafios. A reabilitação através de ambientes virtuais potencializa a plasticidade cerebral quando associada a estímulos motores, sensoriais e visuais. As ações pretendidas por meio das metas motoras somadas ao feedback visual resultam em um aumento da taxa de recuperação do paciente [4].

Porém, a perda da função de um membro requer grandes ajustes nas interações com o mundo físico. Uma resposta comum a essa perda após um AVE é o aprendizado de formas compensatórias de movimento, incluindo o uso da mão não parética. Também são observadas alterações compensatórias na coordenação dos movimentos de ambos os membros, bem como do membro parético em conjunto com o tronco $[12,15]$. Algumas formas de compensação são óbvias, mas outras são sutis o suficiente para passar despercebidas na ausência de medidas comportamentais sensíveis, tornando-as facilmente confundidas [8]. Além do deslocamento excessivo do tronco [10], compensações motoras durante o alcance também foram descritas em termos de rotações axiais excessivas do tronco $[9,17]$, movimentos escapulares [14] e sinergia flexora, onde o movimento conjunto do ombro e do cotovelo permite ao paciente levantar o braço trazendo-o de volta ao corpo em simultâneo [11].

Um método amplamente utilizado para análises quantitativas de movimentos é o estudo baseado em modelos musculoesqueléticos. Esses modelos biomecânicos fornecem uma estrutura para a integração de dados anatômicos e fisiológicos, permitindo uma ampla gama de estudos no controle neuromuscular. Modelos dos membros superiores, por exemplo, têm sido usados como uma forma de melhor compreensão do controle neuromuscular na reabilitação de pacientes após um AVE [5].

Um ambiente virtual adaptável, somado a uma análise biomecânica musculoesquelética, pode se mostrar bastante conveniente durante a reabilitação. Além de permitir que os indivíduos realizem 
exercícios terapêuticos específicos de forma agradável e motivadora, o aprendizado de movimentos compensatórios inapropriados e prejudiciais para sua plena reabilitação pode ser evitado $[7,16]$.

\section{SOLUÇÃO PROPOSTA}

Sob tais perspectivas, esta proposta de tese de doutorado aborda um sistema integrado, cujo objetivo é auxiliar na reabilitação motora de pacientes com hemiparesia nos membros superiores, decorrente de um AVE. Para tanto, O sistema proposto é composto por um ambiente virtual e um dispositivo robótico, altamente personalizáveis, que permitirá ao terapeuta ajustar as condutas da reabilitação mediante a evolução clínica do paciente.

Além disso, pretende-se mapear a atividade musculoesquelética do membro afetado através de um modelo tridimensional. Este modelo detectará variações biomecânicas de modo a fornecer um feedback motor que pode ser utilizado para análise e correção de movimentos compensatórios durante a execução do treinamento. Uma rede de sensores inerciais também será implementada com o intuito de capturar e comparar a dinâmica de movimento e alterações anatômicas do membro.

Assim, pretende-se avaliar a adequação do sistema desenvolvido como ferramenta de apoio nos processos de reabilitação e auxílio ao terapeuta na definição de protocolos de reabilitação mais específicos para cada indivíduo.

\section{RESULTADOS PRELIMINARES}

O ambiente virtual encontra-se em desenvolvimento, o qual é composto por uma floresta virtual, onde o paciente controla uma harpia, podendo movê-la livremente em diferentes direções e em graus adequados de liberdade, a depender dos objetivos e desafios a serem cumpridos. Inicialmente, foram desenvolvidas três fases no ambiente. Cada fase possui objetivos diferentes, trabalhando movimentos específicos, como flexão e extensão do cotovelo e coordenação motora e visuoespacial. Também é possível configurar e customizar cada parâmetro conforme a necessidade terapêutica de cada paciente.

Para a interface de controle, está sendo construído um protótipo de uma plataforma robótica para suporte assistido, que dispõe de um mecanismo composto por dois motores acionados por cabos que atuam em um plano cartesiano. Por meio desse mecanismo, é possível fornecer um feedback de assistência de movimentos. $\mathrm{O}$ ambiente virtual foi integrado à plataforma de força utilizando a interface de comunicação desenvolvida, onde a movimentação do manipulador nos eixos X e Y é refletida nos movimentos da harpia.

\section{CONCLUSÃO}

O uso de um sistema integrado pode ser de grande valia para o processo de reabilitação, principalmente quando ela é realizada utilizando-se de ambientes altamente ajustáveis e que respeitem as limitações individuais, oferecendo ao fisioterapeuta ferramentas de análise de evolução e resultados para tratamento. O dispositivo robótico e o ambiente virtual somados a um modelo musculoesquelético completo que responda conforme o rastreamento de movimentos do membro afetado, podem conseguir dar respostas importantes associadas a compensação de movimentos que afetam o processo de reabilitação.
Almeja-se a utilização desse sistema como método de reabilitação futura, visto que ele oferece uma prática de exercícios mais eficientes frente a indivíduos mais estimulados. Desse modo, torna-se imprescindível proporcionar a prescrição de protocolos de tratamentos fisioterapêuticos individualizados que aumentem a motivação, interação e aderência dos pacientes à reabilitação.

\section{AGRADECIMENTOS}

Os autores agradecem à Coordenação de Aperfeiçoamento de Pessoal de Nível Superior (CAPES) pelo apoio financeiro ao projeto.

\section{REFERÊNCIAS}

[1] Ministério da Saúde (BR). 2013. Diretrizes de atenção à reabilitação da pessoa com acidente vascular cerebral. https://bvsms.saude.gov.br/bvs/publicacoes/ diretrizes atencao reabilitacao acidente vascular cerebral.pdf

[2] David A Greenberg, Michael J Aminoff, and Roger P Simon. 2014, pp. 488. Neurologia clínica, 8rd ed. Artmed, Porto Alegre.

[3] Arthur Clifton Guyton. 2006. Tratado de fisiologia médica. Elsevier Brasil.

[4] Megan Heenan, Robert A Scheidt, Douglas Woo, and Scott A Beardsley. 2014. Intention tremor and deficits of sensory feedback control in multiple sclerosis: a pilot study. Journal of NeuroEngineering and Rehabilitation 11, 1 (2014), 170. DOI: 10.1186/1743-0003-11-170.

[5] John W Krakauer. 2006. Motor learning: its relevance to stroke recovery and neurorehabilitation. Current Opinion in Neurology 19, 1 (Feb. 2006), 84-90. DOI: 10.1097/01.wco.0000200544.29915.cc.

[6] Peter Langhorne, Robert Wagenaar, and Cecily Partridge. 1996. Physiotherapy after stroke: More is better? Physiotherapy Research International 1, 2 (June 1996), 75-88. DOI: 10.1002/pri.6120010204.

[7] Kate Laver, Stacey George, Susie Thomas, Judith E. Deutsch, and Maria Crotty. 2012. Virtual Reality for Stroke Rehabilitation. Stroke 43, 2 (Feb. 2012). DOI: 10.1161/strokeaha.111.642439.

[8] Mindy F. Levin, Jeffrey A. Kleim, and Steven L. Wolf. 2008. What Do Motor "Recovery" and "Compensation" Mean in Patients Following Stroke? Neurorehabilitation and Neural Repair 23, 4 (Dec. 2008), 313-319. DOI: 10.1177/1545968308328727.

[9] Mindy F. Levin, Eliane C. Magdalon, Stella M. Michaelsen, and Antonio A. F. Quevedo. 2015. Quality of Grasping and the Role of Haptics in a 3-D Immersive Virtual Reality Environment in Individuals With Stroke. IEEE Transactions on Neural Systems and Rehabilitation Engineering 23, 6 (Nov. 2015), 1047-1055. DOI: 10.1109/tnsre.2014.2387412.

[10] Mindy F. Levin, Stella M. Michaelsen, Carmen M. Cirstea, and Agnès Roby-Brami. 2002. Use of the trunk for reaching targets placed within and beyond the reach in adult hemiparesis. Experimental Brain Research 143, 2 (Jan. 2002), 171-180. DOI: 10.1007/s00221-001-0976-6.

[11] R. E. Lrvine. 1972. Movement Therapy in Hemiplegia: A Neurophysiological Approach. The Gerontologist 12, 2 Part 1 (June 1972), 205-208. DOI: 10.1093/geront/12.2_part_1.205a.

[12] Hirofumi Nakayma, Henrik Stig Jørgensen, Hans Otto Raaschou, and Tom Skyhøj Olsen. 1994. Compensation in recovery of upper extremity function after stroke: The Copenhagen Stroke Study. Archives of Physical Medicine and Rehabilitation 75, 8 (Aug. 1994), 852-857. DOI: 10.1016/0003-9993(94)90108-2.

[13] Susan B O'sullivan, Thomas J Schmitz, and George D Fulk. 2017, pp. 1688. Fisioterapia: avaliação e tratamento, $6 r d$ ed. Editora Manole.

[14] Johanna V. G. Robertson, Nicolas Roche, and Agnès Roby-Brami. 2012. Influence of the side of brain damage on postural upper-limb control including the scapula in stroke patients. Experimental Brain Research 218, 1 (Feb. 2012), 141-155. DOI: 10.1007/s00221-012-3014-y.

[15] Edward Taub, Gitendra Uswatte, and Victor W. Mark. 2014. The functional significance of cortical reorganization and the parallel development of CI therapy. Frontiers in Human Neuroscience 8 (June 2014). DOI: 10.3389/fnhum.2014.00396.

[16] M. Thornton, S. Marshall, J. McComas, H. Finestone, A. McCormick, and H. Sveistrup. 2005. Benefits of activity and virtual reality based balance exercise programmes for adults with traumatic brain injury: Perceptions of participants and their caregivers. Brain Injury 19, 12 (Jan. 2005), 989-1000. DOI: $10.1080 / 02699050500109944$.

[17] Joost van Kordelaar, Erwin E. H. van Wegen, and Gert Kwakkel. 2012. Unraveling the interaction between pathological upper limb synergies and compensatory trunk movements during reach-to-grasp after stroke: a cross-sectional study. Experimental Brain Research 221, 3 (July 2012), 251-262. DOI: 10.1007/s00221012-3169-6.

[18] Derick T. Wade. 1993. Measurement in neurologic rehabilitation. Current Opinion in Neurology 6, 5 (Oct. 1993), 778-784. DOI: 10.1097/00019052-199310000-00017. 